\title{
Attentional Demand Varies During a Blind Navigation Pathway In Young And Older Adults
}

\author{
Yves Lajoie $^{1, *}$, Nicole Paquet ${ }^{2}$ and Renée Lafleur ${ }^{1}$ \\ ${ }^{1}$ School of Human Kinetics University of Ottawa Ontario, Canada K1N 6N5 \\ ${ }^{2}$ School of Rehabilitation Sciences, University of Ottawa Ontario, Canada K1H 8 M5
}

\begin{abstract}
This study sought to investigate the attentional demands while performing a blind navigation task in young and elderly subjects. Fourteen subjects of age 20 to 32 years and 10 subjects of age 62 to 80 years participated in the experience. Blinded navigation task consisted of visually identifying and then walking blindly towards a target 8 meters ahead. To measure attentional demands during navigating, participants were asked to respond to auditory signals by saying "top" as quickly as possible without altering their gait. Reaction times were longer in the older adults and approaching the target for the two groups. Navigation measures (traveled distance, angular deviation and body rotation) were significantly larger in older than young adults. Interestingly, the dual-task leads both groups to walk significantly further which brought the young subjects closer to the target and elderly subjects further from the target.
\end{abstract}

Keywords: Aging, attentional demands, blind navigation, gait, reaction time

\section{INTRODUCTION}

The concurrent performance of a cognitive and a locomotor task is known to become more difficult with aging. A systematic review of dual-task studies in older adults indicate that reduced gait speed and performance decrement in the cognitive task are larger in old than in young adults [1]. Among explanations for these age-related changes in dual-task performances, it has been proposed that aging involves general slowing in neural processing [2], impairment in management and coordination of multiple tasks [3] and performance decrement in tasks of increased complexity [4]. However, the generally accepted concept is that during dual-tasks attention resources are shared between the primary and the concurrent secondary tasks, presuming that each task require attention [5]. If the attention capacity of an individual is exceeded when executing the two tasks concurrently, then performance on one or both tasks should decrease [6]. Indeed, old adults were found to have longer reaction times than young adults when responding verbally to an auditory cue during walking [7]. This is in spite of a slower gait speed and shorter steps that provided older participants with a better postural stability during walking.

Spatial navigation is a functional outcome of walking. Specifically it is any displacement in space to reach a destination. Aging is known to affect spatial navigation likely because of progressive decline in vision and kinesthetic sensation, as well as in sensory-motor and cognitive functions [8]. For instance, navigation is challenged in older adults by the frequent distractions encountered in daily life [9-11]. When vision was reduced, old adults made direction errors that increased even

*Address correspondence to this author at the School of Human Kinetics University of Ottawa Ontario, Canada K1N 6N5; Tel: (613) 562 5800; Fax: (613) 562-5149; E-mail: Ylajoie@uottawa.ca more when navigation was executed in conditions of divided attention [12].

The challenging task of navigating without vision to reach a previously seen target has extensively been studied in young healthy adults [13]. The attentional requirement of blind navigation is one aspect that has been investigated with dual-task paradigms. Results indicated that the precision in reaching the target, gait speed and performance on the counting backwards task were significantly decreased in dual-task compared to when navigation and the cognitive task were performed separately $[14,15]$.

In contrast to this knowledge in young adults, very little is known on the impact aging has on the attentional demands of navigation without vision towards a previously seen target. This is important to investigate as it relates to safety of displacements in low lighting or in darkness, as when one walks from the bedroom to the bathroom at night for example. Our main research question is whether the task of navigating without vision requires more attention in older adults than in younger adults. To answer this question, we used a concurrent reaction time (RT) task during blind navigation to test whether performance on one or both tasks are affected in young and older participants, in comparison to when tasks are executed separately and then, whether the possible decrease in performance during dual-task is worse in older participants. Our hypotheses are that RT should be longer during dual-task than in the baseline sitting condition, and longer in older than in young participants.

Our second question is whether the attention required to perform blind navigation is uniform or not along the navigation path. We hypothesize that RT should increase in both older and young participants when they are approaching the target because of the additional attention needed when they wonder if they are now near the target, and to prepare for stopping. 
Table 1. The Table Shows the Exact Sequence of RTs (Represented by X) that was Used for Each Participant in the Dual-Task Condition. Trial \#7 was a Catch Trial with no RT

\begin{tabular}{|c|c|c|c|c|}
\hline Trial \# & Interval \#1 & Interval \#2 & Interval \#3 & Interval \#4 \\
\hline \hline 1 & & & $x$ & $x$ \\
\hline 2 & & $x$ & $x$ & $x$ \\
\hline 3 & $x$ & $x$ & $x$ & $x$ \\
\hline 4 & & $x$ & $x$ & $x$ \\
\hline 5 & $x$ & $x$ & $x$ & $x$ \\
\hline 7 & $x$ & $x$ & $x$ & $x$ \\
\hline 10 & $x$ & $x$ & $x$ \\
\hline
\end{tabular}

The first aims of the study were to establish the impact of aging on the attentional demand of a blind navigation task with the use of a dual-task that includes a RT task. Our second aim isto describe in both groups of participants variations in the attentional demand along the blind navigation pathway.

\section{MATERIALS AND METHODOLOGY}

\section{Participants}

Fourteen young and 10 older adults participated in this study. Young participants were 23.7 years old on average (standard deviation (SD) of 2.9 years). Eight of the 14 young participants were females. Older participants were 72.6 years old on average (SD of 4.3 years). Seven of the 10 older participants were females. Both groups were similar in height and weight. All participants were living in the community. They were included if they were in good health and reported no impairment of their control of balance and gait. Older subjects were excluded if they had a score of 25 or less on the Mini-Mental State Examination. All subjects signed an informed consent form approved by the local Ethics Committee.

\section{Procedures}

Experimental set up. On the floor of a gymnasium, a start line and a target located 8 meters away were made with tape. On the side of this walkway, three small pieces of tape indicated the 2, 4 and 6-meter distances from the start line. This was done to visually divide the 8-meter walkway into four 2-meter intervals: Interval \#1: 0-2 meters, interval \#2: 2 4 meters, interval \#3: 4-6 meters and interval \#4: 6-8 meters. Participants, however, were not aware of these divisions.

Navigation task. Participants were standing behind the starting line. They were instructed to look at the target located 8 meters ahead and then, after 5-10 seconds of looking, to wear opaque goggles. They then got a "go" signal from the experimenter and walked towards the target without vision, while walking at a comfortable pace. Their task was to walk until they believed they had arrived at the target. They then stopped and the experimenter marked the position of their feet on the floor. For security purpose, an assistant was closely and silently following participants while they were navigating without vision. Opaque goggles were a pair of modified ski goggles that completely removed vision. At the end of each trial, participants remained blindfolded, were seated in a wheelchair and were returned to the start line by an assistant. This was done to prevent them from knowing their final position relative to the target and correct the trajectory in subsequent trials. In addition, marks of feet position were made of clear adhesive tape and were not visible from the start line. Participants could not see them when they were preparing for a next navigation trial.

Baseline RT task. Participants were sitting and wore opaque goggles. A speaker was located close to them, as well as an MP3 digital audio recorder. The experimenter manually triggered the delivery of auditory stimuli, consisting of $1000 \mathrm{~Hz}, 50 \mathrm{~ms}$ in length. The stimulus sounded like a 'beep'. Participants were instructed to respond verbally 'top' as quickly as possible after each stimulus. Ten stimuli were delivered at the beginning of the testing session and ten at the end. Stimuli were sent at random intervals ranging from 1.5 to $5 \mathrm{sec}$.

Dual-task. While they were navigating towards the target, participants had to verbally respond 'top' as quickly as possible to auditory stimuli, as in the baseline sitting condition. They were instructed that the navigational task was the primary task and the RT task, the secondary task. The assistant who was closely following participants during their blind navigation wore a portable speaker device in a pouch strapped around the waist, and was holding the MP3 digital audio recorder. The assistant was always slightly behind, and on the participants' right side. Thus, the origin of auditory stimuli relative to participants was at similar and constant location. The experimenter triggered auditory stimuli when participants were within specific distance intervals to the target. Walkway intervals were 2 meters long. The stimulus was sent when the participant was within the interval. The experimenter was sending the stimulus approximately midway in the interval. Two or three stimuli were given per trial, except for one trial without stimulus (trial \#7: catch trial). Only one stimulus per interval was given. A sequence of stimulus delivery was determined beforehand, to ensure that subjects would not anticipate the stimuli. Table 1 shows the sequence of stimuli that was used for all participants. 


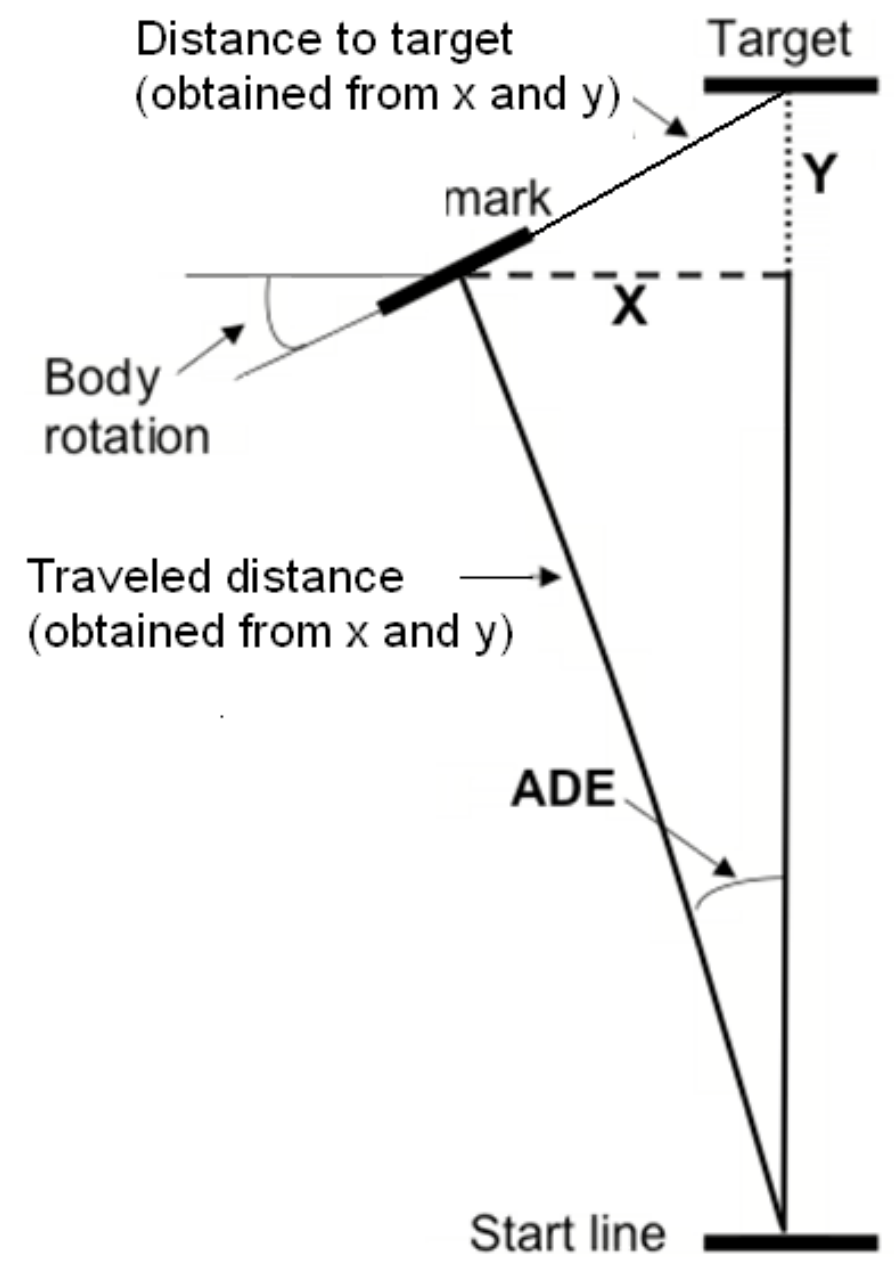

Fig. (1). Illustration of the start line, a target located at $800 \mathrm{~cm}$ from the start line and a footmark. $\mathrm{X}$ and $\mathrm{Y}$ coordinates are shown, as well as angular deviation (ADR), body rotation and distance to target.

\section{Data Collection}

All participants went through the experimental protocol in the same order. Ten baseline sitting RT trials were done first. Then, participants performed five trials of the navigation task, followed by five trials of the dual-task, and again, five navigation trials and five dual-task trials. Finally, 10 more baseline sitting RT trials were done. The testing session was about 30 minutes in duration.

In order to obtain four measures of navigation precision from feet position marks, two distances were measured: the lateral ( $\mathrm{x}$ ) and the longitudinal distance $(\mathrm{y})$ in reference to the straight-line trajectory, as shown in Fig. (1). These distances were measured in centimeters using a measuring tape and a one-meter ruler. In addition, body rotation (BR) was measured in degrees using a protractor aligned with the angle of the floor mark. Traveled distance was obtained with the following formula: Traveled distance $=\sqrt{ }\left(x^{2}+(y+800)^{2}\right)$. Angular deviation (ADE) was obtained with trigonometric calculation: $\operatorname{ADE}\left({ }^{\circ}\right)=\operatorname{ATAN}[\mathrm{X} /(\mathrm{Y}+800)]$. ADE towards the right of the subject was considered as a positive deviation, and the left, a negative one. For BR, clockwise rotation was a positive rotation and counterclockwise rotation, a negative one. Distance to target was obtained with the following formula: DT $(\mathrm{cm})=\sqrt{ }\left(\mathrm{x}^{2}+\mathrm{y}^{2}\right)$. RTs were determined as the time lapse in milliseconds between the onset of the auditory stimulus and the beginning of the verbal response that were both recorded on the MP3 recorder. These data were transferred onto an IBM laptop computer. Onsets were obtained by visual inspection of traces with the use of Audacity software that allows zooming of traces.

\section{Statistical Analysis}

Baseline RTs at the start of the session were compared to those at the end with a t-test. No significant effect of time was found. Therefore, we pooled the start and end sitting RT and used the mean value for further analyses.

Separate two-way ANOVAs (group X condition) were performed on mean traveled distance, ADE, BR and distance to target. A one-way ANOVA (group) was performed on speed. A two-way ANOVA (group $\mathrm{X}$ condition and intervals) was done on mean RTs. Post hoc (Tukey) analyses were used to determine the location of the differences when the ANOVA revealed significant differences with a $\mathrm{p}$ value smaller or equal to 0,05 . 


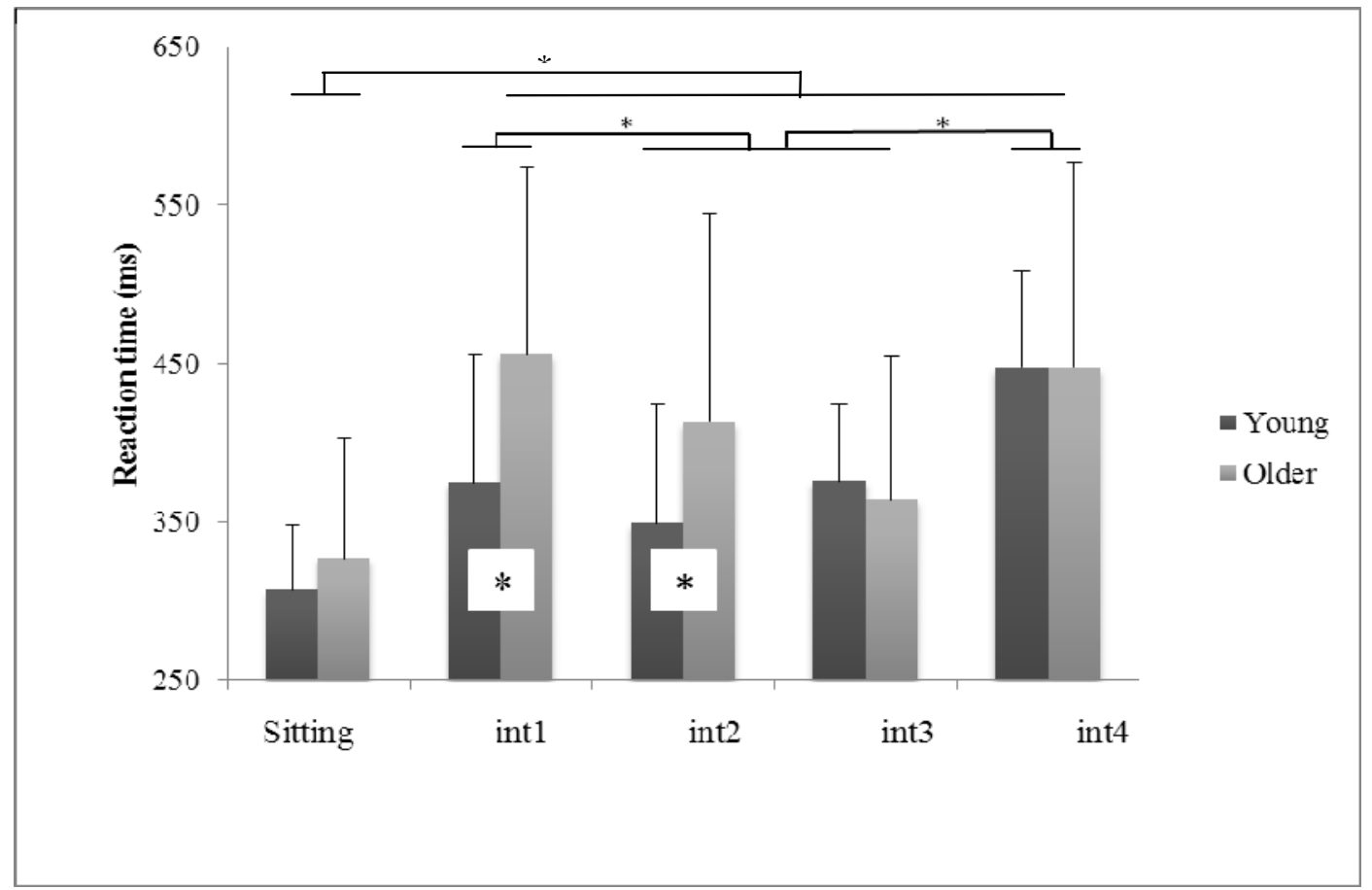

Fig. (2). Means $+1 \mathrm{SD}$ of RT (msec) during sitting and dual-task intervals (Int1=0-2 m; Int2=2-4 m; Int3=4-6 m; Int4=6-8 m). (*p<0.01).

\section{RESULTS}

\section{Reaction Time}

Fig. (2) illustrates RT results. The two-way ANOVA revealed no significant effect of group $\mathrm{F}(1,21)=0.90, \mathrm{p}>0.05$, but a significant effect of condition $F(4,84)=23.87, p<0.001$ and a significant interaction group $X$ condition $F(4,84)=4.01$, $\mathrm{p}<0.01$. For both groups, baseline sitting RTs were significantly shorter than during navigation $(\mathrm{p}<0.05)$. Compared to baseline RTs, dual-task RTs were significantly longer at all four intervals in young participants, and at intervals 1, 2 and 4 in older participants. There were also significant group differences in RTs among intervals. In young participants, RT at interval 4 was significantly longer than in the other intervals $(\mathrm{p}<0.05)$. In the older participants, RT at interval 1 was significantly longer than at intervals 2 and 3 . RT at interval 4 was significantly longer than at intervals 2 and $3(p<0.05)$.

\section{Navigation Performance}

Results revealed no significant effect of groupfor speed $(\mathrm{F}(1,22)=3.69, \mathrm{p}>=0.05)$. On average, young participants were walking slightly faster $(1.1 \mathrm{~m} / \mathrm{s})$ than the older participants $(0.88 \mathrm{~m} / \mathrm{s})$ but the difference was found not significant. As well, distance to target (Fig. 3A) was not significantly different between group and condition $(\mathrm{F}(1,22)=3.57, \mathrm{p}>0.05, \mathrm{~F}(1,22)=0.22, \mathrm{p}>=0.05$ respectively $)$. However, the two-way ANOVA revealed a significant interaction group $\mathrm{X}$ condition $(\mathrm{F}(1,22)=11.42, \mathrm{p}<0.001)$ indicating that small changes in distance to target due to condition were not the same between the two groups. In dual-task, young participants got closer to the target than during the navigation task, while older participants stopped further to the target. For traveled distance, a significant effect of group $(F(1,22)=25.17, p<0.001)$ and a significant effect of condition were found $(\mathrm{F}(1,22)=39.41, \mathrm{p}<0.001)$, and a significant group $x$ condition interaction $(F(1,22)=4.74$, p $<0.05$ ). Fig. (3B) shows that traveled distance was significantly shorter during navigation than dual-task and significantly shorter in young than old participants. The interaction is explained by a greater increased of the traveled distance in the dual-task condition for the old participants. ADE results revealed a significant effect of group $(\mathrm{F}(1,22)=4.12, \mathrm{p}<0.05)$, no significant effect of condition $(\mathrm{F}(1,22)=0.01, \mathrm{p}>0.05)$ and no interaction $(\mathrm{F}(1,22)=1.91$, $\mathrm{p}>0.05)$. BR results revealed a significant effect of group $(F(1,22)=6.39, p<0.05)$, no significant effect of condition $(\mathrm{F}(1,22)=0.80, \mathrm{p}>0.05)$ and no interaction $(\mathrm{F}(1,22)=0.07$, $\mathrm{p}>0.05)$. Older participants had a larger ADE and BR than younger participants (Fig. 3C and 3D).

\section{DISCUSSION}

Our first finding is that blind navigation requires more attention than baseline sitting in both young and older participants. This is consistent with previous demonstration that as balance and locomotion tasks are more challenging, the attentional demand increases in parallel [16-18]. Blind navigation had previously been found to interfere with a cognitive task, as the rate of backward counting was significantly decreased during dual-task compared to baseline sitting [15]. Aging was found to further accentuate the increased attentional demand of balance and gait tasks [7]. Sparrow, Bradshaw, Lamoureux \& Tirosh (2002) found that when walking with eyes open to reach a specific target, older participants had similar RTs than young adults when using a auditory stimulus. Such absence of main group effect is also what we found in our study.

We showed that aging has an impact on the variation in attention during navigation. A significant group $\mathrm{x}$ condition interaction indicated that RT changes during navigation were different in older compared to young participants, while RT in baseline sitting was not different between the two groups. In young participants, a significant increase in RT occurred 


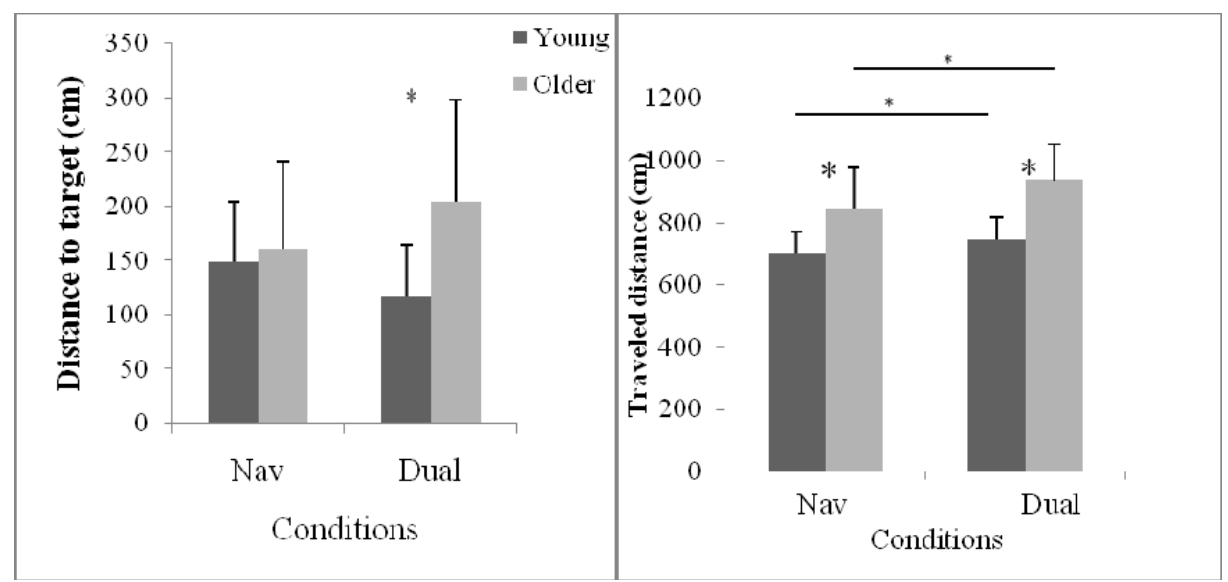

C

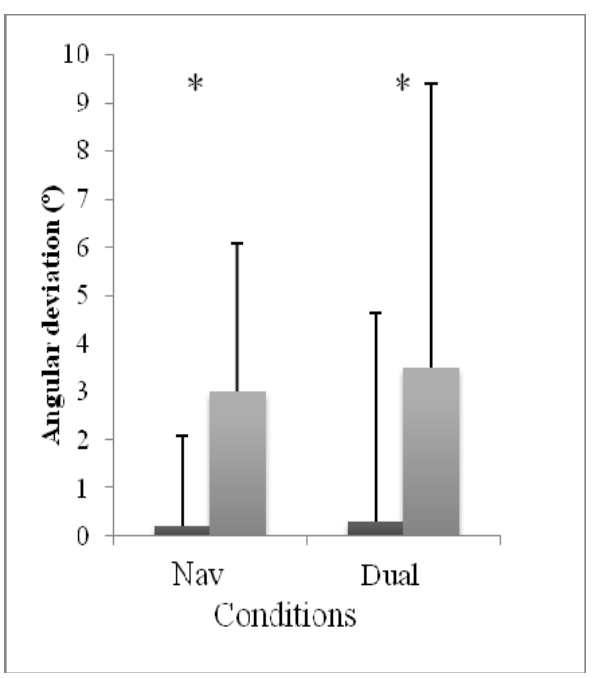

$\mathrm{D}$

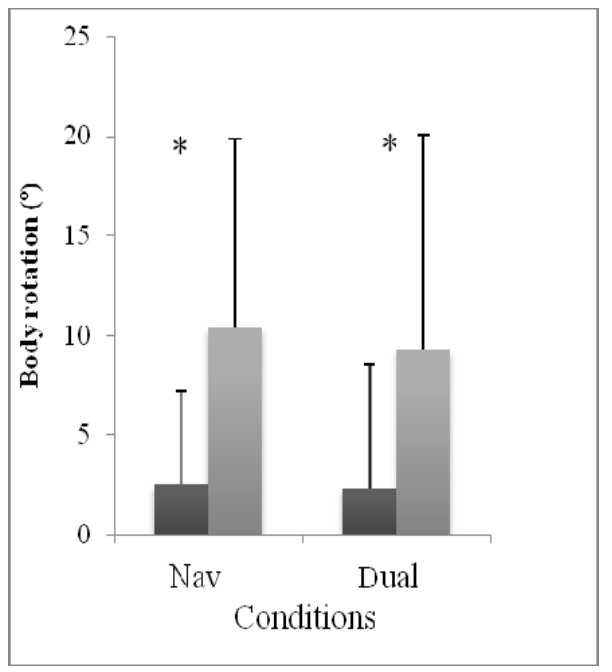

Fig. (3). (A) Mean distance to target $(\mathrm{cm})+1 \mathrm{SD} ;(\mathbf{B})$ Mean traveled distance $(\mathrm{cm})+1 \mathrm{SD} ;(\mathbf{C})$ Mean angular deviation (degrees) $+1 \mathrm{SD} ;(\mathbf{D})$ Mean body rotation (degrees) $+1 \mathrm{SD}$ for the young and older group during navigation and dual-task conditions. $\left({ }^{*} \mathrm{p}<0.01\right)$

at interval 4, when they were approaching the target. Such increase in attention nearby the target had previously been described for walking with eyes open $[16,19]$. In contrast, older participants had longer RTs at intervals 1 and 4 , indicating that attention was higher at both gait initiation and target approach. It supports previous report of longer RTs at the beginning of walking in old adults [19], and suggests that with aging, gait initiation requires additional attention. This is likely due to the dynamic balance challenge associated with moving from the stable standing position to the unstable action of walking [20]. Older participants also had longer RTs at target approach. Thus, both young and older participants had less attention available for the RT task at that moment, probably because they were aware of now being close to the target and were preparing for stopping.

Our results suggest that RTs interfered, at least in part, with blind navigation, in spite of differences in modalities of the two tasks; RT is an auditory-verbal task and navigation is a spatial orientation-walking task. Consistent with this, a recent systematic review indicates that RT has equivalent interference with walking parameters than other types of cognitive tasks [1]. Such interference between heterogeneous modalities is better explained by the capacity-sharing theory of information processing that states that when two attentiondemanding tasks are performed simultaneously, performance of one of both tasks would deteriorate if the attention capacity is exceeded [21].

In general, navigation performance was not affected by dual-task with the exception of traveled distance that was significantly longer during dual-task than in navigation alone. This is in line with an increase in distance traveled during blind navigation when a concurrent secondary backward counting task was executed [22]. These authors proposed that the transformation of self-velocity in space to estimate distance traveled might be a variable affected by the secondary task, as well as errors in time, i.e. time integration. Later, they suggested that spatial and temporal integration could rely on the same neural substrate [14]. If participants are progressively estimating their distance to target from the time they think it may take to get there, the RT task probably temporarily interrupts this cognitive process. The associated delay in time estimation may explain that all participants walked further without noticing it. 
As expected, navigation performance was better in young than older participants. Significant group effect was found for traveled distance, ADE and BR. Larger direction errors during dual tasks involving blind navigation had previously been identified in older adults [23]. These authors suggested that age-related deficits in the encoding of body rotation information from the vestibular system leads to a decline in the accuracy of path integration. Age-related differences in path integration performance are likely related to the amount of cognitive resources, including speed of processing and working memory.

Interestingly, navigation precision as measured with distance to target was not affected by aging. However, the significant group $\mathrm{x}$ condition interaction shows that the two groups behaved differently in dual-task. Young participants got slightly closer to the target, while older participants ended up further to target. This could be explained by the behavior of the young participants who undershoot the target while the older participants overshoot the target. By adding the secondary task, both groups walked further.

\section{CONCLUSION}

Our findings and those of previous navigation and RT studies suggest that in daily walking, increased attention demands would reduce the resources available for other concurrent tasks. In demanding gait tasks such as walking in darkness, competing distractions associated with secondary stimulus could lead to declines in gait performance and increases in the risk of falling. Allocation of large attentional resources while navigating with low vision may reduce the response time to a hazard and has also safety implications if more errors are made on location.

\section{CONFLICT OF INTEREST}

The authors confirm that this article content has no conflicts of interest.

\section{ACKNOWLEDGEMENTS}

This project was financed by the Canadian Institutes of Health Research.

\section{REFERENCES}

[1] Al-Yahya E, Dawes H, Smith L, Dennis A, Howells K, Cockburn J. Cognitive motor interference while walking: a systematic review and meta-analysis. Neurosci Biobehav Rev 2011; 35: 715-28.
[2] Birren JE. Translations in gerontology: from lab to life. Psychophysiology and speed of response. Am Psychol 1974; 29: 808-15.

[3] Korteling JE. Effects of skill integration and perceptual competition on age-related differences in dual-task performance. Human Fact 1991; 33: 35-44.

[4] McDowd JM, Craik FI. Effects of aging and task difficulty on divided attention performance. J Exp Psychol 1988; 14: 267-80.

[5] Kerr B, Condon SM, McDonald LA. Cognitive spatial processing and the regulation of posture. J Exp Psychol 1985; 11: 617-22.

[6] Shumway-Cook A, Woollacott M. Attentional demands and postural control: the effect of sensory context. J Gerontol Ser A 2000; 55: M10-16.

[7] Lajoie Y, Teasdale N, Bard C, Fleury M. Upright standing and gait: Are there changes in attentional requirements related to normal aging? Exp Aging Res 1996; 22: 185-98.

[8] Craik FIM,Salthouse TA. The handbook of aging and cognition. Second edition. New Jersey: Erlbaum 2000.

[9] Tolman EC. Cognitive maps in rats and man. Psychol Rev 1948; 55: 189-208.

[10] Trullier O, Wiener SI, Berthoz A, Meyer JA. Biologically based artificial navigation systems: Review and prospects. Prog Neurobiol 1997; 51: 483-544.

[11] Verghese J, Buschke H, Viola L, et al. Validity of divided attention tasks in predicting falls in older individuals: a preliminary study. $\mathrm{J}$ Am Geriat Soc 2002; 50: 1572-6.

[12] Broman AT, West SK, Muñoz B, Bandeen-Roche K, Rubin GS, Turano KA. Divided visual attention as a predictor of bumping while walking: the Salisbury Eye Evaluation. Investig Ophthalmol Visual Sci 2004; 45: 2955-60.

[13] Loomis JM, Klatzky RL, Golledge RG. Navigation without vision: Basic and applied research. Optometry Vis Sci 2001; 78: 282-9.

[14] Glasauer S, Stein A, Günther AL, Flanagin VL, Jahn K, Brandt T. The effect of dual tasks in locomotor path integration. Ann N Y Acad Sci 2009; 1164: 201-5.

[15] Paquet N, Lajoie Y, Rainville C, Sabagh-Yazdi F. Effect of navigation direction on the dual-task of counting backward during blind navigation. Neurosci Lett 2008; 442: 148-51.

[16] BardyBG, Laurent $M$. Visual cues and attention demand in locomotor positioning. Percept Motor Skills 1991; 72: 915-26.

[17] Lajoie Y, Teasdale N, Bard C,Fleury M. Attentional demands for static and dynamic equilibrium. Exp Brain Res 1993; 97: 139-44.

[18] Teasdale N, Bard C, LaRue J, Fleury M. On the cognitive penetrability of postural control. Exp Ageing Res 1993; 19: 1-13.

[19] Sparrow WA, Bradshaw EJ, Lamoureux E, Tirosh O. Ageing effects on the attention demands of walking. Hum Mov Sci 2002; 21: $961-72$.

[20] Chang H, Krebs DE. Dynamic balance control in elders: gait initiation assessment as a screening tool. Arch Phys Med Rehabil 1999; 80: 490-94.

[21] Tombu M, Jolicoeur P. A central capacity sharing model of dualtask performance. J Exp Psychol Hum Percept Perform 2003; 29: 3-18.

[22] Glasauer S, Schneider E, Grasso R, Ivanenko YP. Space-time relativity in self-motion reproduction. J Neurophysiol 2007; 97: 451-61.

[23] Allen G, Kirasic K,Rashotte M. Aging and path integration skill: Kinesthetic and vestibular contributions to wayfinding. Percept Psychophys 2004; 66: 170-9. 